\title{
Avaliação de Dispositivos de Rastreamento em uma Rede LoRaWAN no Contexto de Cidades Inteligentes
}

\begin{abstract}
Marcos V. R. da Silva ${ }^{1}$, José E. de Souza ${ }^{1}$, Thiago Vinney Oliveira Almeida ${ }^{1}$, Álvaro R. C. Souza ${ }^{1}$, Fabio A. Spanhol ${ }^{1}$, Edson T. de Camargo ${ }^{1}$

${ }^{1}$ Universidade Tecnológica Federal do Paraná (UTFPR) - Câmpus Toledo Rua Cristo Rei, 19 - Vila Becker - CEP 85902-490 - Toledo - PR - Brasil

Resumo. $O$ rastreamento de veículos de frotas está entre as principais aplicações de cidades inteligentes. Este trabalho realiza uma avalição de quatro dispositivos rastreadores - dois comerciais e dois programados - que fazem uso de uma rede LoRaWAN para enviar suas geolocalizações coletadas através de sensores GPS. A avaliação dos dispostivos faz parte do desenvolvimento de uma solução de rastreamento de caminhões da coleta seletiva de lixo em parceria com a Universidade e o Município. O comportamento de cada dispositivo é analisado ao percorrer 3 regióes urbanas representativas. Os resultados incluem uma análise quantitativa e qualitativa para apresentar a eficiência dos dispositivos e dados sobre de intensidade do sinal da rede e relação sinal-ruído.
\end{abstract}

\begin{abstract}
Fleet vehicle tracking is among the main applications for smart cities. This work evaluates four tracking devices - two commercial and two programmed - that make use of a LoRaWAN network to send their geolocations collected through GPS sensors. The evaluation of the devices is part of the development of a truck tracking solution for selective garbage collection in partnership with the University and the Municipality. The behavior of each device is analyzed when moving through 3 representative urban regions. The results include a quantitative and qualitative analysis to present the efficiency of the devices, as well as data on network signal strength and signal-to-noise ratio.
\end{abstract}

\section{Introdução}

Embora ainda não haja consenso sobre quais características ou requisitos definam cidades inteligentes (smart cities), algumas definições são centradas na importância do uso da tecnologia [Sánchez-Corcuera et al. 2019]. Ou seja, para alguns autores, a inclusão de tecnologias de informação e comunicação é essencial para construir uma cidade inteligente. Nesse sentido, a combinação de tecnologias de sensoriamento, redes sem-fio de longo alcance e infraestrutura computacional para processamento de grandes volumes de dados heterogêneos possibilitam elaborar soluções inteligentes e escaláveis para lidar com os desafios dos grandes centros urbanos [Santana et al. 2017].

No geral, uma cidade inteligente compreende domínios de aplicação diversos como monitoramento ambiental [Addabbo et al. 2019], transporte público [James and Nair 2017] e rastreamento de frotas [Farooq and Pesch 2018]. Sistemas de rastreamento e monitoramente normalmente empregam um sistema de geolocalização global, como o GPS (Global Positioning System), para alimentar as aplicações com coordenadas geográficas, seja de veículos, pessoas ou objetos [Andrade and Yoo 2019]. Através de um dispositivo GPS é possível, por exemplo, 
rastrear um veículo em movimento dentro de uma cidade e armazenar o histórico do seu percurso. Aplicações podem estimar a velocidade de um veículo, alertar quando um veículo se aproxima ou se distancia de uma origem e mesmo se desvia de uma rota programada. Assim, gestores públicos podem fiscalizar a rota e tomar medidas para, por exemplo, evitar congestionamentos ao acompanhar em tempo real a posição de veículos.

Um requisito central para qualquer aplicação em cidades inteligentes é a capacidade de transmitir seus dados através de uma rede de comunicação [Yaqoob et al. 2017]. Em uma cidade inteligente, uma rede de comunicação de dados não é utilizada para conectar exclusivamente pessoas, mas qualquer objeto [Zanella et al. 2014]. O conceito de Internet das Coisas - ou IoT (Internet of Things) - é empregado para definir objetos conectados a Internet capazes de gerar informação e serem representados no mundo virtual [Bardyn et al. 2016]. Tais objetos possuem características particulares quanto ao uso de uma rede: (i) enviam pequenas quantidades de dados periodicamente; (ii) conectam-se diretamente à Internet ou entre si através de um enlace sem fio; (iii) são ligados a uma bateria e/ou painel solar; (iv) podem estar espalhados por áreas de difícil acesso e (v) estão fixos ou em movimento - em um poste ou em um veículo em movimento, por exemplo. Nesse aspecto, destacam-se as redes de baixo consumo de energia e longo alcance, conhecidas como LPWAN (Low Power Wide Area Network) [Centenaro et al. 2016, Guibene et al. 2017].

Uma rede LPWAN permite enviar periodicamente apenas poucos quilobits por segundo (Kbps), mas pode cobrir áreas de até 50 quilômetros em campo aberto. Também proporciona maior vida útil para as baterias dos dispositivos finais (sensores, atuadores) e ocupa faixas de frequência de bandas não licenciadas. Entre as tecnologias e protocolos de LPWAN aplicados em cidades inteligentes destaca-se o padrão aberto LoRaWAN, que opera sobre a tecnologia sem fio LoRa (Long Range) [Andrade and Yoo 2019]. Uma característica importante do padrão LoRaWAN é não cobrar qualquer taxa pela utilização da rede e estimular o próprio cliente a instalar sua infraestrutura de rede. Nesse sentido, a UTFPR/Toledo implantou e avaliou uma rede LoRaWAN para apoiar o desenvolvimento de aplicações para cidades inteligentes [Rossato and Camargo 2019]. Inserida no escopo desse mesmo projeto, uma aplicação de rastreamento de frotas está sendo desenvolvida em parceria com o Município para monitorar em tempo real os caminhões da coleta seletiva de lixo. Dessa forma, entre outras possibilidades, os moradores podem ser notificados via aplicativo de celular sempre que o caminhão de coleta se aproxima de sua residência. O desenvolvimento dessa solução de rastreamento passa pela construção de um protótipo e avaliação da cobertura da rede.

Embora existam trabalhos para avaliar a tecnologia LoRa no Brasil [Ortiz et al. 2018, Ortiz et al. 2019], ainda há carência de implementações práticas em ambientes reais de monitoramento, conforme apontado por [Oliveira et al. 2018]. Logo, o objetivo deste trabalho foi avaliar dispositivos de rastreamento de veículos em uma rede LoRaWAN para futuramente selecionar o dispositivo a ser empregado nos caminhões da coleta seletiva. Especificamente foram avaliados quatro dispositivos: dois comerciais destinados a rastreamento em redes LoRaWAN; um dispositivo com GPS e transmissor LoRa embutido, mas sem a camada LoRaWAN e que foi programado usando a biblioteca LMIC; e outro dispositivo montado com um Arduino Uno, transmissor GPS e um transmissor comercial LoRaWAN. Os quatro dispositivos foram fixados em um veículo, 
simulando um caminhão da coleta seletiva. A avalição ocorreu em três áreas em torno do câmpus da UTFPR, com tamanhos aproximados de $0,34 \mathrm{~km}^{2}, 0,77 \mathrm{~km}^{2}$ e 9,6 km². Os resultados apresentam a eficiência de cada dispositivo nas três áreas. Há também uma avaliação qualitativa, que considera o indicador de intensidade do sinal recebido (Received Signal Strength Indicator - RSSI), relação sinal-ruído (Signal to Noise Ratio - SNR) e fator de espelhamento para as coordenadas recebidas. O custo benefício de cada dispositivo também é apresentado. Apesar do objetivo principal ter sido avaliar os dispositivos em si, é possível estender a análise para o sinal da rede LoRaWAN do câmpus.

Este artigo está organizado da seguinte forma. A Seção 2 apresenta os padrões LoRa e LoRaWAN, incluindo alguns trabalhos relacionados. A Seção 3 descreve os dispositivos analisados e a metodologia de avaliação. A Seção 4 apresenta os resultados obtidos e a Seção 5 a conclusão e trabalhos futuros.

\section{LoRa e LoRaWAN}

A tecnologia de transmissão sem fio LoRa, desenvolvida e patenteada pela empresa Semtech, realiza a modulação de radiofrequência (RF) baseada no espalhamento espectral de chirp (Chirp Spread Spectrum - CSS). Essa técnica é utilizada na comunicação militar e espacial permitindo cobrir longas distâncias e alcançar robustez à interferência [LoRa Alliance 2017]. Uma mensagem LoRa pode ser de uplink ou downlink. A estrutura da mensagem é semelhante em ambos os casos, no entanto, apenas a mensagem de uplink adiciona um código de verificação (CRC) para garantir a integridade da carga útil (PHYPayload).

No Brasil, a faixa de utilização da LoRa é de $915 \mathrm{MHz}$, compreendendo 902 $\mathrm{MHz}$ a $907,5 \mathrm{MHz}$ e $915 \mathrm{MHz}$ a $928 \mathrm{MHz}$. Em 2018 a ANATEL publicou o Ato $n^{o}$ 6.506 que aprova os procedimentos para avaliação da conformidade de equipamentos de radiocomunicação de radiação restrita, permitindo a operação de dispositivos LoRa no território nacional [ANATEL 2018]. Apesar de ser possível utilizar dois padrões de transmissão, o americano e o australiano [LoRa Alliance 2017], este último vem sendo adotado no Brasil. Tal padrão possui 72 canais para uplink e 8 para downlink. No uplink, os canais de 0 a 63 utilizam uma largura de banda de $125 \mathrm{kHz}$ com uma taxa de codificação 4/5, iniciando em 915,2 MHz com incremento linear de $200 \mathrm{kHz}$ até 927,8 MHz. Já os canais de 64 a 71 possuem uma largura de banda de $500 \mathrm{kHz}$ a partir de $915,9 \mathrm{MHz}$, incrementando linearmente de $1,6 \mathrm{MHz}$ até $927,1 \mathrm{MHz}$. No downlink os canais de 0 a 7 possuem uma largura de banda de $500 \mathrm{kHz}$, iniciando em 923,3 MHz com incremento linear de $600 \mathrm{kHz}$ até $927,5 \mathrm{MHz}$.

Para usar a modulação LoRa é necessário configurar três parâmetros no dispositivo final que determinam o bit rate (quantidade de bits por segundo), o tamanho máximo do payload (carga útil de informações) e o air time (tempo em que o pacote está no ar), para permitir um maior alcance ou enviar uma quantidade maior de bits na mensagem:

- Largura de Banda (Bandwidth, BW): pode assumir três valores determinados, 125 $\mathrm{kHz}, 250 \mathrm{kHz}$ ou $500 \mathrm{kHz}$, sofrendo um deslocamento de até $20 \%$ que não influenciará na decodificação [Oliveira et al. 2018];

- Fator de Espalhamento (Spreading Factor, SF): determina o número de chirps necessários para representar um símbolo (um ou mais bits de dados), apresentando 
$2^{S F}$ valores possíveis de 7-12. Isso influencia a duração do um chirp - quanto maior o SF mais energia será usada por bit e maior será o alcance entre transmissor e receptor [Ortiz et al. 2018];

- Taxa de Código (Code Rate, CR): define o número de bits destinados para dados de redundância na mensagem, a fim de realizar a recuperação de erros, definidos entre 1 e 4 com os respectivos valores do CR: 4/5, 4/6, 4/7 e 4/8. Incrementar o CR aumenta a proteção, mas também o tempo do bit no ar [Ortiz et al. 2018].

LoRaWAN é um protocolo aberto de comunicação para redes LoRa gerido pela entidade aberta e sem fins lucrativos LoRa Alliance, que congrega mais de 500 membros espalhados pelo mundo. Uma visão geral da arquitetura de uma rede LoRaWAN é mostrada na Figura 1. Os dispositivos finais (end nodes) são objetos equipados com sensores e/ou atuadores. O gateway conecta os dispositivos na rede LoRaWAN com a Internet através do servidor de rede, o qual gerencia a comunicação dos dispositivos com o servidor de aplicação. O servidor de aplicação exibe as informações vindas dos dispositivos para o usuário final [LoRa Alliance 2017].

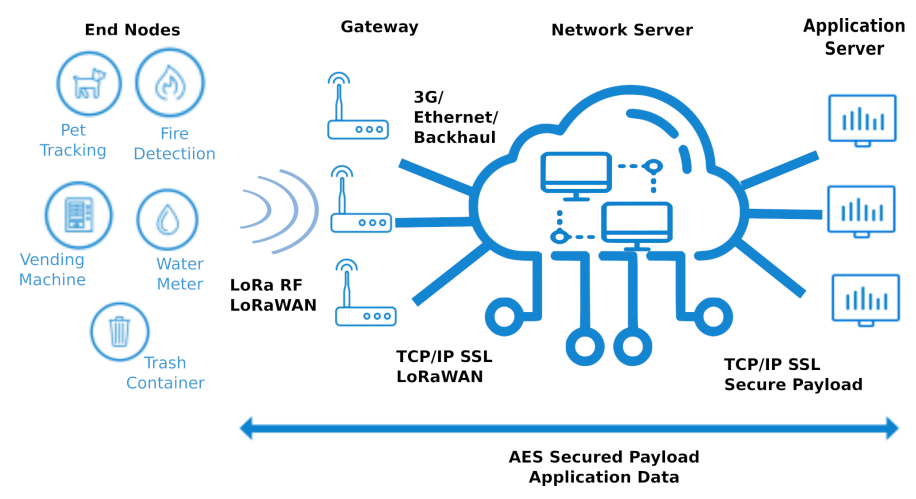

Figura 1. Visão geral da arquitetura LoRaWAN. Baseada em [LoRa Alliance 2017]

Os dispositivos finais podem assumir três configurações: i) Classe A - é a configuração obrigatória nas demais classes e nela o dispositivo final inicia a comunicação com o gateway, realizando uma transmissão e abrindo duas janelas de recepção de dados do gateway; ii) Classe B - a comunicação é iniciada pelo gateway, que determina o momento de transmissão do dispositivo final e na sequência abre duas janelas de recepção e iii) Classe $\mathrm{C}$ - o dispositivo final realiza uma transmissão de dados, abre duas janelas de recepção e mantém uma janela aberta até a próxima transmissão, fazendo com que o dispositivo final necessite de uma fonte constante de energia.

Em segurança, LoRaWAN oferece criptografia em dois níveis: (i) AES-CMAC na camada de rede para o dispositivo final e (ii) AES-CCM* na camada de aplicação para a comunicação entre o servidor de aplicação e os dispositivos finais. Também há dois tipos de autenticação: OTAA (Over-The-Air Activation), mais robusta, chaves trocadas com a rede em sessão de ativação iniciada pelo dispositivo; e ABP (Activation By Personalization), mais simples, com chaves armazenadas diretamente no dispositivo.

\subsection{Rastreamento Através de LoRa/LoRaWAN}

James e Nair [James and Nair 2017] propuseram uma alternativa aos sistemas convencionais de rastreamento de transporte público normalmente baseados em GPS. O modelo 
proposto usa a transmissão sem fio LoRa para se comunicar entre os pontos de paradas de ônibus e uma estação base. A comunicação entre ônibus e pontos se dá através de transmissores de RF. Podevijn et al. [Podevijn et al. 2018] avaliaram a performance da geolocalização LoRa para uma aplicação de rastreamento em uma rede LoRaWAN pública. Segundo os autores, considerar conjuntamente o mapa do percurso e a velocidade do veículo melhora significativamente a acurácia de localização, com erro médio inferior a 75 metros. Visando reduzir os custos, inerentes aos sistemas de rastreamento baseados em tecnologias GSM/GPRS, Hattarge, Kekre e Kothari [Hattarge et al. 2018] propuseram um sistema de rastreamento para ônibus urbanos baseado em GPS e LoRaWAN. No sistema aberto de rastreamento apresentado por Ramli et al. [Ramli et al. 2019], a velocidade e geolocalização de barcos de turistas deslocando-se em um geoparque protegido são acompanhadas em tempo real através de transponders GPS para Arduino.

\section{Materiais e Métodos}

Os quatro dispositivos considerados neste trabalho estão descritos na Tabela 1.

\begin{tabular}{|c|c|c|c|c|}
\hline & RAK7200 & RAK5205 WisTrio & T-Beam & Arduino + Shield \\
\hline Microcontrolador & $\begin{array}{l}\text { ST STM32L073 } \\
\text { Arm Cortex M0 } \\
\text { 32-bit } \\
32 \mathrm{KHz}-32 \mathrm{MHz}\end{array}$ & $\begin{array}{l}\text { ST STM32L1 MCU } \\
\text { Arm Cortex M3 } \\
\text { 32-bit } \\
32 \mathrm{KHz}-32 \mathrm{MHz}\end{array}$ & $\begin{array}{l}\text { TTGO } \\
\text { Tensilica LX6 } \\
\text { 32-bit } \\
240 \mathrm{MHz} \\
\end{array}$ & $\begin{array}{l}\text { Microchip } \\
\text { ATmega 328P-PU } \\
\text { 8-bit } \\
20 \mathrm{MHz}\end{array}$ \\
\hline Memória Flash & $192 \mathrm{~KB}$ & $512 \mathrm{~KB}$ & $4 \mathrm{MB}$ & $32 \mathrm{~KB}$ \\
\hline Memória RAM & $20 \mathrm{~KB}$ & $80 \mathrm{~KB}$ & $520 \mathrm{~KB}$ & $2 \mathrm{~KB}$ \\
\hline Transmissor LoRa & Semtech SX1276 & Semtech SX1276 & Semtech SX1276 & Semtech SX1272 \\
\hline Antena LoRa & interna & $2 \mathrm{dBi}$ & $2,5 \mathrm{dBi}$ & $2,15 \mathrm{dBi}$ \\
\hline Transmissor GPS & Sony-Semicon CXD5603GF & Ublox Max 7Q & Ublox Neo-6M & Ublox Neo-6M \\
\hline Antena GPS & interna & externa & externa & externa \\
\hline Alimentação & bateria recarregável & bateria recarregável & bateria recarregável & externa $5 \mathrm{~V} \mathrm{CC}$ \\
\hline Custo (US\$) & 39.50 & 49.50 & 26.50 & 45.00 \\
\hline
\end{tabular}

Tabela 1. Especificação dos dispositivos avaliados.

Os dispositivos rastreadores comerciais da série Rak7200 e Rak5205 são enddevices normalmente utilizados para prototipação rápida de soluções IoT baseadas em LoRa. Não são programáveis. O dispositivo de rastreamento TTGO T-Beam é um enddevice constituído de uma placa montada em torno de um chip ESP32 de núcleo duplo com adição do módulo LoRa e GPS. Sendo um dispositivo microcontrolado é obrigatório realizar a programação dos seus módulos para realizar as funções necessárias. Arduino é uma plataforma aberta para prototipação eletrônica com hardware expansível através de placas chamadas shields e bibliotecas de software. Neste trabalho foram utilizadas shields GPS e LoRa. Assim como o T-Beam, o Arduino precisa ser programado. O Rak5205 é o mais caro, possivelmente por incluir outros sensores, como temperatura e umidade, que foram desativados durante os experimentos. O T-Beam é o mais barato, com mais memória e velocidade de processamento quando comparado aos demais. Já o Rak7200 é vendido como um rastreador de propósito geral, é pequeno e pode ser fixado na cintura de um pessoa.

Para programar o módulo LoRa, no dispositivo T-Beam, foi usada a biblioteca LMIC, modificada pela MCCI Corporation. Essa biblioteca implementa uma abstração 
de hardware, funcionando como uma máquina de estado e é responsável por realizar a comunicação entre a camada física LoRa e outros dispositivos gateway. Já o módulo NEO-6M foi programado com o auxílio da biblioteca TinyGPS++, usada para realizar o controle de fluxo de dados NMEA, padrão comum entre dispositivos de navegação, incluindo receptores GPS.

\subsection{Metodologia de Avaliação}

Para avaliar a capacidade da utilização da rede LoRa no cenário de rastreamento, e também os dispositivos construídos e programados em relação aos dispositivos comerciais, foram utilizadas três áreas distintas de avaliação, como mostrado na Figura 2. Cada área foi verificada em separado, mesmo que contenham regiões sobrepostas, com os dados coletados sendo obtidos por três passagens em dias diferentes. A coleta foi feita utilizando os quatro dispositivos avaliados, descritos na Tabela 1, sem obstrução por parte do veículo, em velocidade média de $20 \mathrm{~km} / \mathrm{h}$. Tal velocidade foi escolhida para aproximar-se da velocidade média desenvolvida pelos veículos quando realizando efetivamente a coleta segundo a rota estabelecida, descartando a etapa em que tais veículos encaminham-se para o descarte final do lixo coletado empregando velocidades mais elevadas. A opção por uma velocidade constante - em detrimento de simular todas as potenciais paradas inerentes ao processo de coleta - deu-se pelo fato de que a velocidade de deslocamento tende a prejudicar a qualidade do sinal aferido, devido a efeitos bem conhecidos como o deslocamento Doppler.

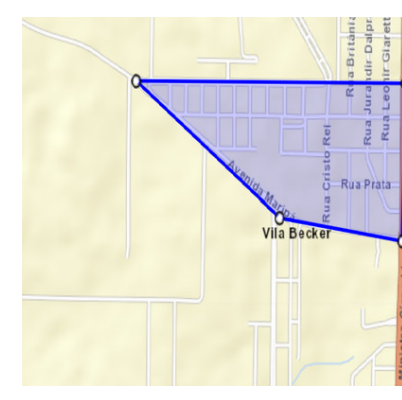

(a) Área 1: $\approx 0,34 \mathrm{~km}^{2}$

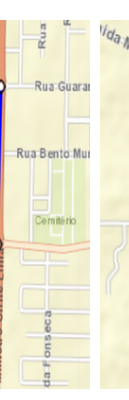

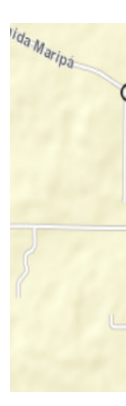

(b) Área 2: $\approx 0,77 \mathrm{~km}^{2}$

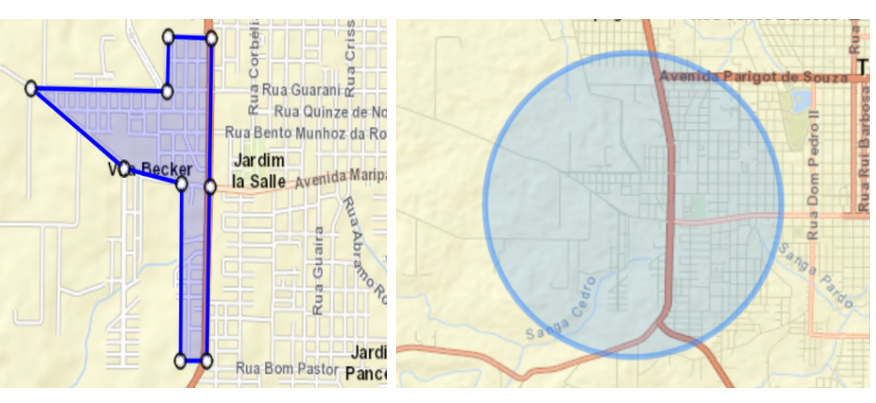

(c) Área 3: $\approx 9,6 \mathrm{~km}^{2}$

Figura 2. Áreas de avaliação.

Os dispositivos foram configurados para fazer o envio de dados a cada 10 segundos, o que, em média, fará com que exista um ponto de medição a cada 5,6 metros. Com isso, é possível avaliar também o impacto de edificações no processo de transmissão, como por exemplo os prédios da própria universidade bem como prédios residenciais e comerciais. Pode haver discordância entre os pontos de GPS entre os quatro dispositivos avaliados, a depender da precisão dos mesmos.

Os dispositos foram avaliados em termos de qualidade de sinal, capacidade de envio de medições e custo-benefício. O primeiro critério pode ser mensurado com o indicador de intensidade do sinal recebido (RSSI) e a relação sinal-ruído (SNR) em cada ponto de medição, com dados fornecidos pela ferramenta TTN Mapper. A capacidade de envio foi medida pela comparação dos pontos de envio entre os dispositivos, verificando em quais locais deixaram de enviar medições e quais razões podem ter levado o dispositivo a não conseguir comunicação, como por exemplo a existência de obstáculos. Por 
fim, o custo-benefício considerou o custo financeiro dos equipamentos em relação às duas métricas de qualidade descritas.

\section{Resultados e Discussão}

Os resultados obtidos foram analisados sob duas perspectivas. A análise quantitativa apresenta a capacidade do dispostivo enviar uma coordenada. Nela os resultados consideram a quantidade de coordenadas recebidas pelo gateway através da ferramenta TTN Mapper para cada dispositivo. A análise qualitativa avalia a qualidade do sinal, ruído e fator de espelhamento para as coordenadas recebidas de cada dispositivo.

\subsection{Análise Quantitativa}

Apesar de definido um intervalo de 10 segundos para cada dispositivo enviar sua geolocalização, na prática isso não se mostrou factível para o T-Beam e para os dispositivos comerciais Rak. Nesse quesito, o Arduino foi o único que conseguiu realmente enviar uma coordenada a cada 10 ou 11 segundos. Tal comportamento pode ser explicado pelo fato do T-Beam estar configurado para usar a classe A, fazendo com que após enviar um pacote ao gateway duas janelas de recebimento de dados de cerca de 1 a 3 segundos sejam abertas. Logo, o intervalo de tempo para cada envio de dado é de aproximadamente 13 segundos. Já nos dois dispositivos Rak, na versão testada do firmware, o comportamento é iniciar uma busca pelo sinal GPS e então realizar o envio efetivo para a rede LoRa. Logo, sempre é verificado um período de tempo superior a 10 segundos para enviar cada coordenada. Além disso, na primeira transmissão, a busca pelo satélite consome cerca de 120 segundos, seguido de uma latência de 2 - 10 segundos para obter a coordenada para então efetivamente transmitir para o gateway e buscar a nova geolocalização. Dessa forma, o tempo de envio de uma coordenada pelos dois Rak foi em média 24 segundos.

As Tabelas 2 e 3 destacam a eficiência de cada dispositivo nas três áreas a partir da quantidade de coordenadas recebidas pelo gateway. A entrada "Cartão SD" refere-se aos dados armazenados pelo Arduino no cartão de armazenamento externo. Tal dispositivo primeiro armazena uma coordenada no seu cartão de memória para então enviá-la para o gateway, o que torna possível conhecer a quantidade de coordenadas que o dispositivo deixou de enviar devido a interrupção de sinal.

A Tabela 2 apresenta os dados das áreas 1 e 2. A coluna "coordenadas" indica a quantidade de coordenadas armazenadas no cartão SD e recebidas pelo gateway a partir dos dispositivos Arduino, T-Beam, RAK5205 e RAK7200. Por exemplo, o cartão SD armazenou 250 coordenadas para a área 1 e 503 coordenadas para a área 2. No entanto, o gateway somente recebeu 212 coordenadas na área 1 e 457 coordenadas na área 2 . Ou seja, 38 coordenadas na área 1 e 46 na área 2 não puderam ser enviadas com sucesso pelo Arduino. A coluna "tempo médio" exibe a quantidade média de segundos que uma coordenada levou para ser recebida pelo gateway. Por exemplo, na área 1 a ferramenta TTN Mapper registrou 1 coordenada a cada 12 segundos para o Arduino e a cada 33 segundos para o RAK7200. Lembrando, como dito no início desta seção, que o RAK7200 somente consegue de fato enviar um nova coordenada a cada 24 segundos. Para a área 2 o Arduino enviou com sucesso uma coordenada a cada 11 segundos e o RAK7200 a cada 37 segundos. Para o RAK7200 isso significa que muitos pacotes foram perdidos.

A coluna "coordenadas previstas" considera o tempo efetivo no qual um dispostivo consegue enviar uma coordenada, ou seja, 10 segundos para o Arduino, 13 segundos para 
o T-Beam e 24 segundos para os Rak. A partir do tempo do percurso estima-se a quantidade de coordenadas que de fato deveriam estar armazenadas no cartão SD e recebidas pelo gateway. Sendo assim, para a área 1, por exemplo, estima-se que 254 coordenadas deveriam estar presentes no cartão de memória do Arduino e que 253 coordenadas deveriam ter sido recebidas pela TTN Mapper através do Arduino. Para o T-Beam, 195 coordenadas deveriam ter sido concretamente enviadas. Já os dispositivos RAK5205 e RAK7200 deveriam ter enviado 105 e 106 coordenadas, respectivamente.

A eficiência de cada dispositivo é uma razão entre as coordenadas obtidas e as coordenadas previstas. O cartão SD não apresentou uma eficiência de $100 \%$ porque uma coordenada foi armazenada entre 10 e 11 segundos (e não 10, conforme desejado). Para as áreas 1 e 2, o T-Beam apresentou a melhor eficiência, embora tenha enviado menos coordenadas para a rede quando comparado com o Arduino. Eficiência aqui representa a capacidade do dispositivo enviar com sucesso o maior número de suas coordenadas previstas. $\mathrm{Na}$ área 1, o Arduino alcançou uma eficiência próxima a do RAK5205. Já o RAK7200 apresentou a pior eficiência, 71,73\%. Na área 2, o Arduino apresentou eficiência superior aos Rak. Por sua vez, os dispositivos RAK5205 e RAK7200 tiveram eficiência de 68,08 $\%$ e $64,97 \%$, respectivamente. A área 1, possivelmente por ser próxima do gateway apresentou a eficiência dos dispositivos acima de $70 \%$. A área 2, por possuir pontos mais afastados, teve a eficiência dos dispositivos reduzida, em particular para os Rak.

\begin{tabular}{lcccccccc}
\hline & \multicolumn{2}{c}{ Coordenadas } & \multicolumn{2}{c}{ Tempo Médio (s) } & \multicolumn{2}{c}{ Coordenadas Previstas } & \multicolumn{2}{c}{ Eficiência } \\
\cline { 2 - 8 } & área 1 & área 2 & área 1 & área 2 & área 1 & área 2 & área 1 & área 2 \\
\cline { 2 - 8 } Cartão SD & 250 & 503 & 10 & 10 & 254 & 512 & $98,46 \%$ & $98,22 \%$ \\
Arduino & 212 & 457 & 12 & 11 & 253 & 512 & $83,79 \%$ & $89,21 \%$ \\
T-Beam & 186 & 347 & 14 & 15 & 195 & 362 & $95,50 \%$ & $95,90 \%$ \\
RAK5205 & 90 & 115 & 28 & 35 & 105 & 169 & $85,82 \%$ & $68,08 \%$ \\
RAK7200 & 76 & 133 & 33 & 37 & 106 & 205 & $71,73 \%$ & $64,97 \%$ \\
\hline
\end{tabular}

Tabela 2. Comportamento dos dispostivos nas Áreas 1 e 2.

A área 3, apresentada na Tabela 3, devido a sua extensão foi dividida em três percursos, denominados percurso 1, 2 e 3 . Para a área 3 apresentamos somente as coordenadas recebidas pela ferramenta TTN Mapper e a eficiência do dispositivo em cada percurso. O percurso 2 é o mais próximo do gateway. O percurso 1 está mais ao norte e o percurso 3 mais a leste em relação ao gateway, porém ambos pertencem a uma região mais distante do gateway. Verifica-se que os dispositivos apresentaram eficiências distintas a depender do percurso realizado. Em geral, o T-Beam foi o de melhor eficiência. $\mathrm{O}$ Arduino oscilou entre $25 \%$ de eficiência para os percursos 1 e 3, e $70 \%$ de eficiência para o percurso 2. Ou seja, os dados mostram que o Arduino se saiu melhor próximo ao gateway. Já os Rak, no geral, tiveram a pior eficiência para a área 3.

\subsection{Análise Qualitativa}

As Figuras 3 - 5 foram obtidas com a ferramenta TTN Mapper, a partir dos pontos recebidos pelo gateway. Como a área 1 está contida na área 2, os resultados da Figura 4 podem ser usados em conjunto aos da Figura 3 para análise da área 1. A coloração dos pontos refere-se à potência do sinal recebido, incluindo tanto o RSSI quanto a SNR. Como a quantidade de pontos dos dispositivos Rak foi bastante inferior aos dispositivos T-Beam 


\begin{tabular}{lcrccrr}
\hline & \multicolumn{2}{c}{ Percurso 1 } & \multicolumn{2}{c}{ Percurso 2 } & \multicolumn{2}{c}{ Percurso 3 } \\
\cline { 2 - 6 } & Coordenadas & Eficiência & Coordenadas & Eficiência & Coordenadas & Eficiência \\
\cline { 2 - 6 } Cartão SD & 944 & $98,00 \%$ & 247 & $98,37 \%$ & 1021 & $94,72 \%$ \\
Arduino & 325 & $25,07 \%$ & 151 & $70,40 \%$ & 262 & $25,90 \%$ \\
T-Beam & 550 & $68,31 \%$ & 204 & $67,37 \%$ & 413 & $69,04 \%$ \\
RAK5205 & 60 & $15,71 \%$ & 31 & $24,60 \%$ & 94 & $24,87 \%$ \\
RAK7200 & 36 & $6,73 \%$ & 13 & $9,32 \%$ & 24 & $5,98 \%$ \\
\hline
\end{tabular}

Tabela 3. Área 3 segmentada em três diferentes percursos.

e Arduino, apenas os dois últimos terão os pontos mostrados em relação às áreas de cobertura. Os pontos de todos os dispositivos serão considerados nas análises sobre fator de espalhamento e CDF (Cumulative Distribution Function) de RSSI e SNR.

$\mathrm{Na}$ área 1, as Figuras 3a-3b mostram que tanto o T-Beam quanto o Arduino foram capazes de registrar uma quantidade satisfatória de pontos. Porém, no canto superior esquerdo da área, há uma região cujos pontos coletados pelo Arduino não foram recebidos pelo gateway. Isso não acontece na Figura 4, que contém a mesma região, o que pode indicar que essa região está no limiar da capacidade de transmissão do Arduino.

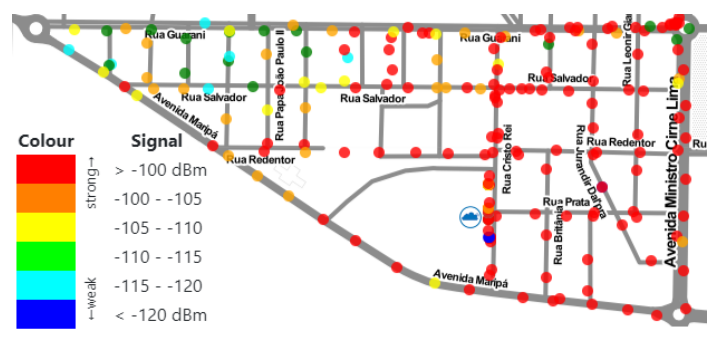

(a) TTGO T-Beam

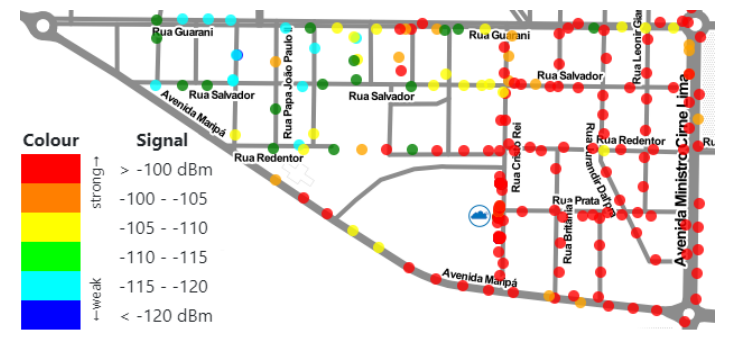

(b) Arduino com shield GPS

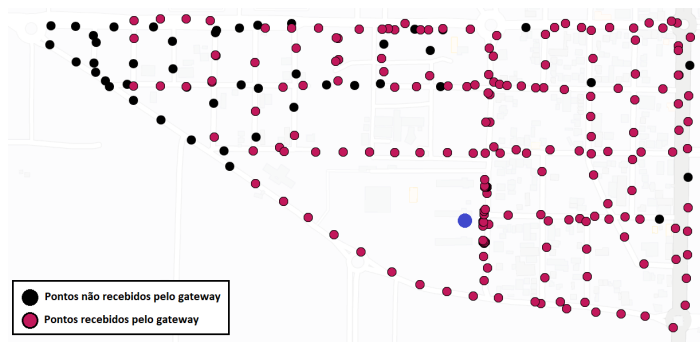

(c) Pontos não recebidos (preto) e recebidos (violeta) para Arduino

Figura 3. Coletas obtidas através do TTN Mapper para cobertura da Área 1.

Como o Arduino registra em cartão de memória os pontos que foram preparados para transmissão, é possível fazer uma comparação para verificar se realmente houve tentativa de envio. Esse resultado é mostrado na Figura 3c, na qual os pontos pretos indicam pontos que foram enviados, e os pontos violetas indicam os que foram recebidos pelo gateway, coletados a partir do TTN Mapper. A área comentada anteriormente registra diversos pontos que não foram recebidos, enquanto o T-Beam recebeu pontos nessa região - não necessariamente a mesma quantidade, pois a própria análise quantitativa mostrou diferença nos intervalos de amostragem. 
Já analisando a área 2, percebe-se pela Figura 4 que ambos os dispositivos apresentaram uma cobertura satisfatória, mesmo em regiões mais distantes. Para a região inferior da área 2, uma explicação plausível para a cobertura dá-se por ser uma área pouco construída e com capacidade de comunicação com linha de visada (LoS) em relação ao gateway. Já na área superior direita, existem alguns poucos prédios, mas a região ainda é dominada por construções residenciais de pequeno porte.

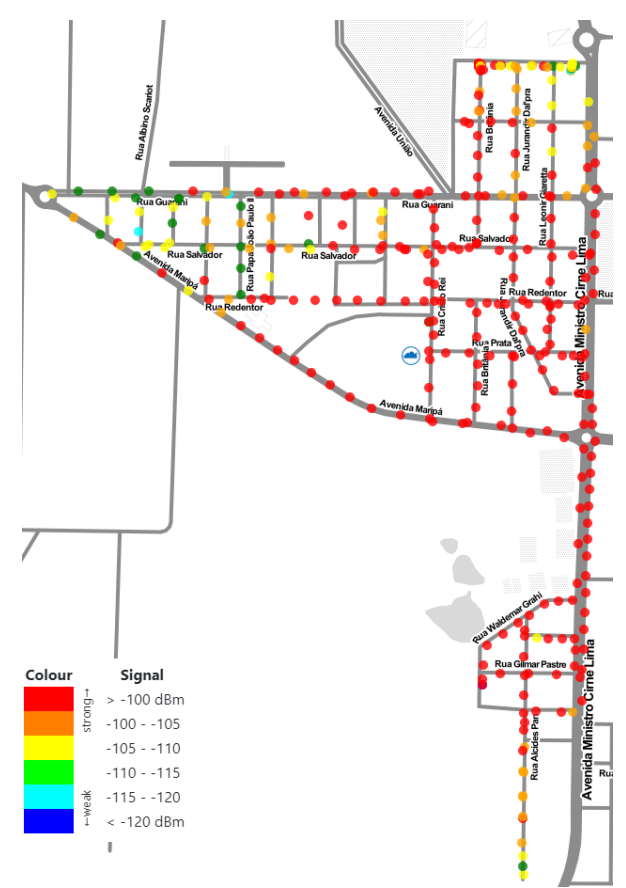

(a) TTGO T-Beam

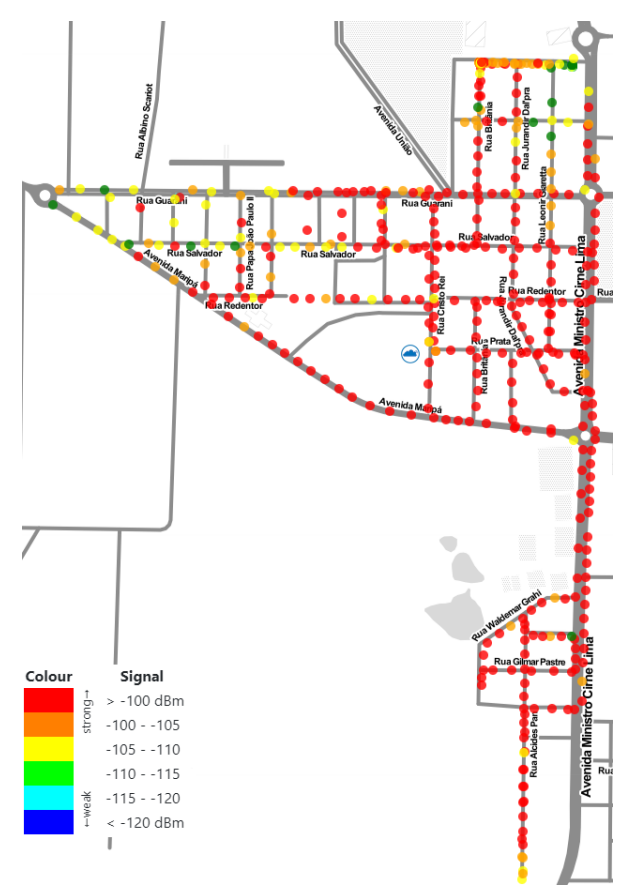

(b) Arduino com shield GPS

Figura 4. Coletas obtidas através do TTN Mapper para cobertura das Áreas 1 e 2.

Por fim, a área 3, apresentada na Figura 5, é a região que apresenta os resultados mais interessantes. Considerando as regiões central e inferior em relação ao gateway, percebe-se que a cobertura, em termos de pontos, é bastante similar, e até com certa vantagem ao Arduino. Porém, nas demais regiões da área 3, a recepção de pontos enviados pelo Arduino mostra-se bastante inferior ao T-Beam. Além da indicação visual na comparação das duas áreas, percebe-se com o auxílio da Figura 5c que há uma grande quantidade de pontos que foram enviados, porém não recebidos pelo gateway. A análise de mapas topográficos mostrou que algumas dessas regiões estão situadas após alguma elevação de relevo, além de serem, no geral, mais habitadas e densamente construidas. Mas, ainda assim, a comparação entre os dispositivos mostra que o T-Beam foi capaz de enviar dados - mesmo os Rak mostraram-se bastante limitados nessas regiões. Note-se, porém, que comparando a Figura 5a com os pontos de coleta do Arduino na Figura 5c, há uma região na área superior esquerda que não foi coberta pelo T-Beam.

Uma das possibilidades para algumas falhas de cobertura ocorre pela elevação atual do gateway, instalado no topo de uma edificação de 4 andares da UTFPR. Para mitigar essa limitação já identificada, a Prefeitura Municipal de Toledo disponibilizou outras edificações mais elevadas para instalação de novos gateways e o estudo de viabilidade de tais locais será um trabalho futuro. 


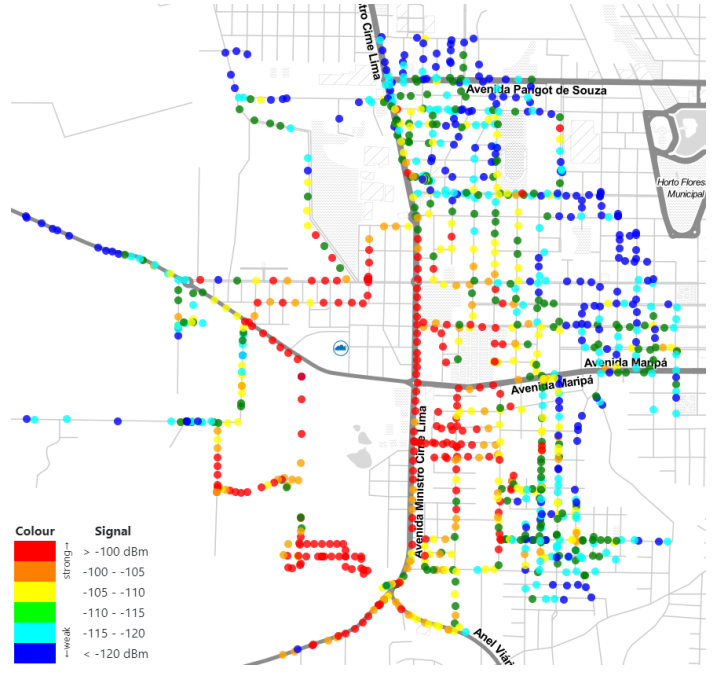

(a) TTGO T-Beam

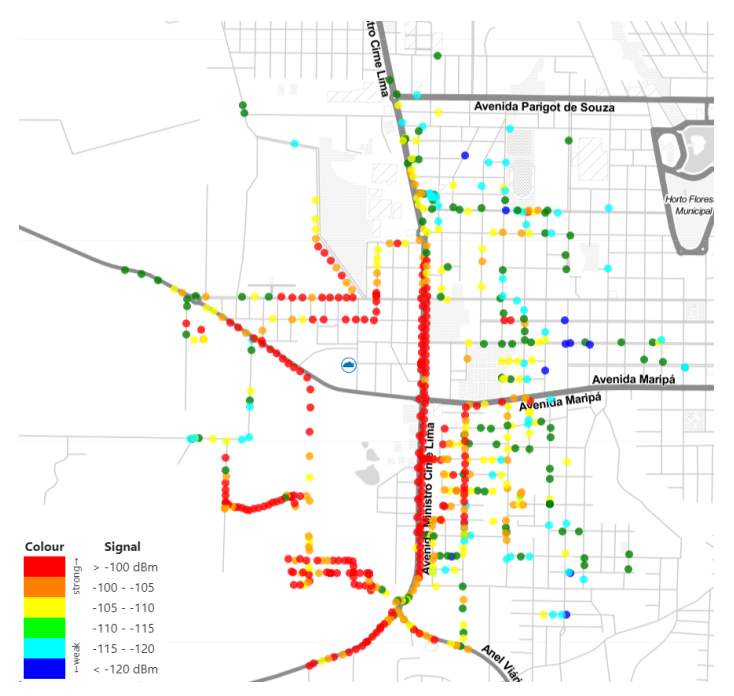

(b) Arduino com shield GPS

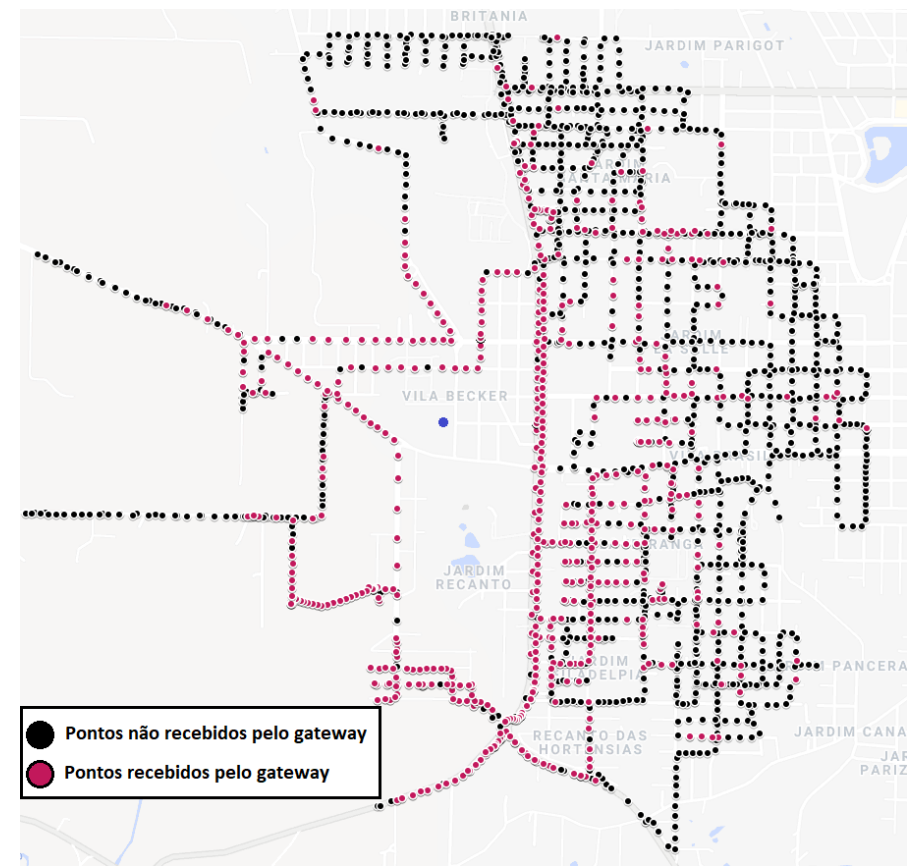

(c) Pontos não recebidos (preto) e recebidos (violeta) para Arduino

Figura 5. Coletas obtidas através do TTN Mapper para cobertura da Área 3.

Outra possibilidade de investigação dá-se pela análise dos dados dos pacotes recebidos, em busca de algo que diferencie os resultados do T-Beam em relação aos demais dispositivos. Um dos parâmetros mantidos pelo TTN Mapper é a sequência de espalhamento (SF) utilizada pelos dispositivos para o envio, juntamente com a largura de banda escolhida. Larguras de banda menores, aliadas a SFs mais elevados, são úteis para ter-se uma transmissão de dados mais confiável, com a penalização de reduzir a taxa de dados. A Figura 6 mostra o SF escolhido pelos dispositivos T-Beam e Arduino nos pontos coletados e recebidos pelo gateway na área 3, plotados de acordo com as coordenadas de latitude e longitude recebidas nos pacotes. Percebe-se que o Arduino majoritariamente adotou SF 7, que é um dos fatores mais curtos, enquanto que o T-Beam adotou uma gama 
mais diversificada de valores. Nas áreas com poucos pontos recebidos do Arduino, principalmente na região superior direita, o T-Beam adotou valores de SF entre 10 e 12 o que o auxiliou a ter mais pontos recebidos. O dispositivo RAK5205 teve comportamento similar ao Arduino, operando com fatores entre 7 e 9, enquanto o RAK7200 utilizou os fatores entre 7 e 12, porém em sua maioria utilizou os fatores entre 7 e 10.
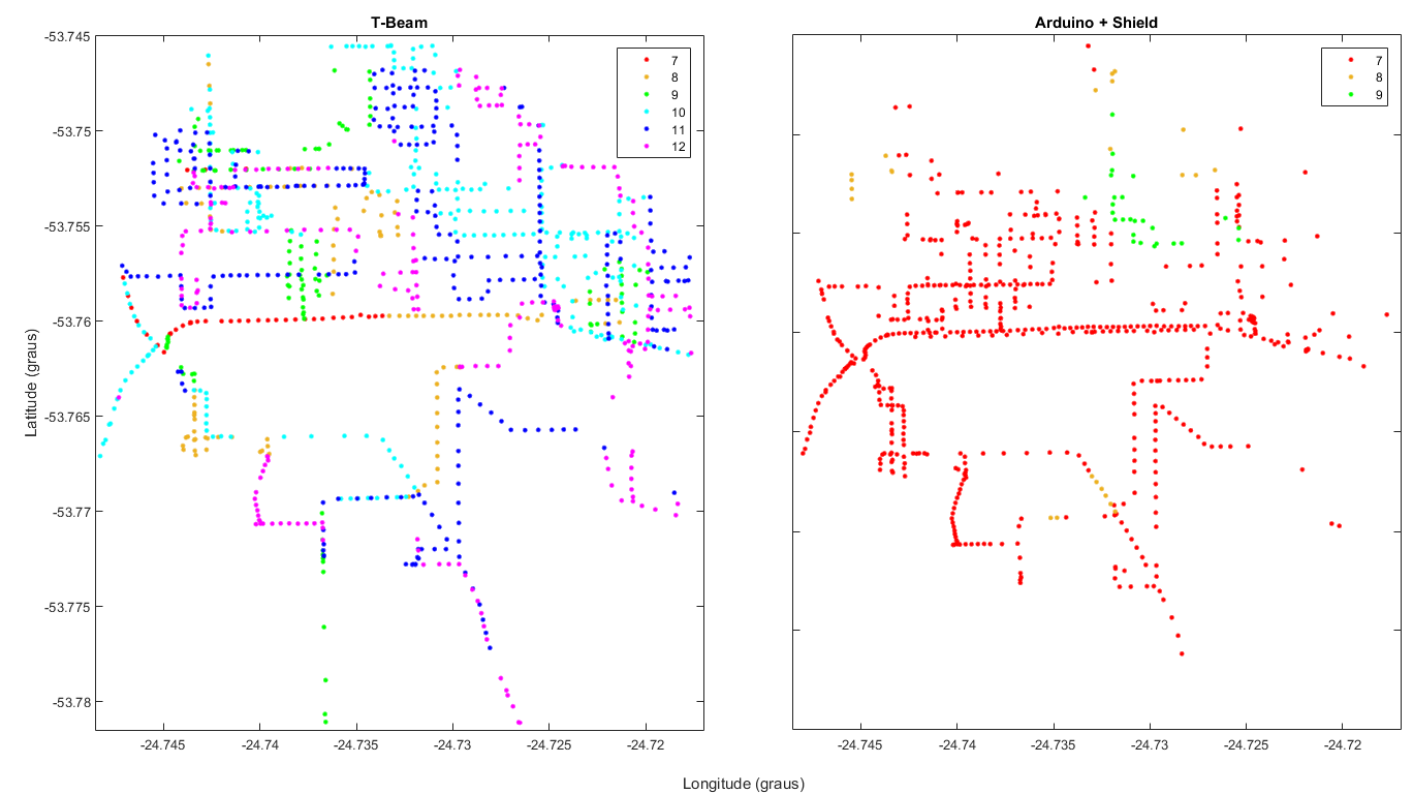

Figura 6. SFs utilizados nos pontos recebidos pelo gateway para o T-Beam (esq.) e Arduino (dir.), de acordo com a latitude e longitude dos pontos.

Uma possível vantagem do dispositivo T-Beam é a utilização da biblioteca LMIC, que possui mecanismos internos de ajuste do fator de espalhamento, mesmo com a ferramenta ADR (Adaptive Data Rate) não ativada. Seguindo a recomendação da documentação de não-utilização do ADR em nós móveis, a presença de um modo de ajuste sem o ADR fez com que o T-Beam se adaptasse melhor aos diversos ambientes, cobrindo uma área superior àquela coberta pelo Arduino e pelos dispositivos RAK.

Por fim, na Figura 7 mostram-se as distribuições de probabilidade empíricas (CDF) para SNR e RSSI. Percebe-se que a curva de distribuição de SNR do T-Beam está mais à esquerda, uma vez que ele foi capaz de coletar pontos mais distantes. O mesmo ocorre com a curva de RSSI. A SNR pode variar em pontos de mesma RSSI, o que justifica observar as duas curvas. Percebe-se também que a curva do RAK7200 possui mais pontos em baixa SNR (lembrando que cada um coletou uma quantidade de pontos diferentes), e uma das causas que pode contribuir para esse comportamento é a utilização de uma antena LoRa interna, enquanto os outros dispositivos utilizam antenas externas. Em termos de RSSI, os dispositivos são bastante equilibrados, com o T-Beam recebendo mais pontos com menor RSSI e o Arduino recebendo mais pontos com maior RSSI (proporcionalmente aos respectivos pontos). 


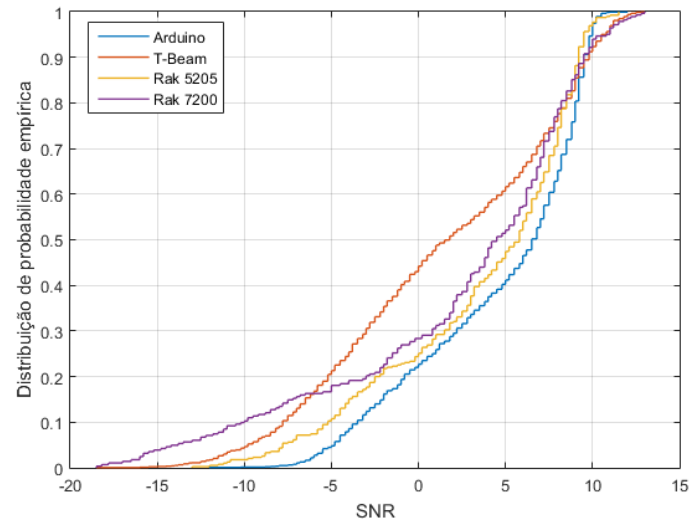

(a) SNR

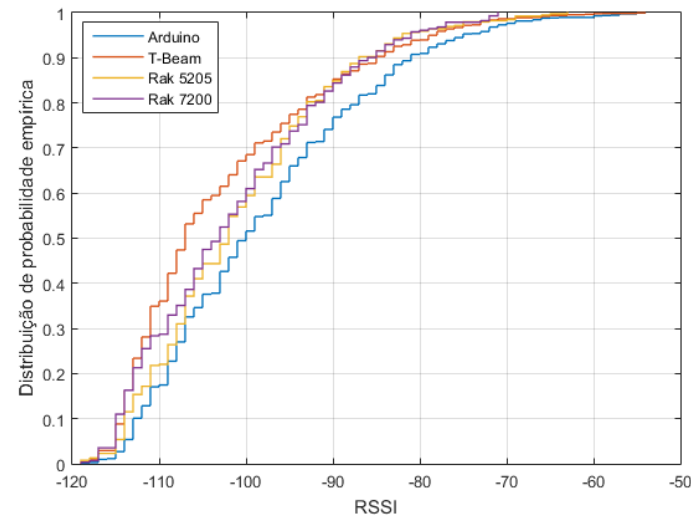

(b) RSSI

Figura 7. Curvas de distribuição de probabilidade empíricas para SNR e RSSI.

\section{Considerações Finais}

Este trabalho realizou uma análise quantitativa e qualitativa de quatro dispositivos de geolocalização que utilizam uma rede LPWAN para enviar seus dados enquanto se movem em três áreas urbanas representativas. A análise realizada serviu principalmente para: a) compreender o comportamento de cada dispositivo móvel sob a rede LoRaWAN; b) entender como as características da rede podem influenciar na coleta de dados móveis e c) subsidiar o desenvolvimento de uma solução de rastreamento para a frota de coleta seletiva de lixo do Município de Toledo. A análise quantitativa demonstrou que o dispositivo T-Beam apresentou a melhor eficiência. Já a análise qualitativa verificou que o T-Beam foi capaz de variar o fator de espalhamento enquanto movimentava-se e que isso pode ter contribuído para seu bom desempenho. Sendo assim, o T-Beam, programado com a biblioteca LMIC, apresentou o melhor custo-benefício dentre os dispostivos avaliados.

De forma geral, os resultados demostraram que coberturas mais abrangentes foram obtidas pelos dispositivos programáveis. As soluções comerciais mostraram-se limitadas possivelmente devido a seus firmwares. Em trabalhos futuros destacam-se as seguintes possibilidades: a) avaliar o consumo energético dos dispositivos em diferentes cenários de configuração; b) determinar um fator de espalhamento ideal que possa otimizar a redução do consumo energético maximizando a intensidade do sinal e a área de cobetura; c) implementar uma estratégia para que os dispositivos enviem seus dados geolocalizados ao detectar um bom sinal da rede e d) planejar a estrutura de gateways para cobrir de forma satisfatória todo o Município de Toledo.

\section{Referências}

Addabbo, T., Fort, A., Mugnaini, M., Parri, L., Pozzebon, A., and Vignoli, V. (2019). Smart sensing in mobility: a lorawan architecture for pervasive environmental monitoring. In 2019 IEEE RTSI.

ANATEL (2018). Ato $n^{\circ}$ 6506, de 27 de agosto de 2018.

Andrade, R. O. and Yoo, S. G. (2019). A comprehensive study of the use of lora in the development of smart cities. Applied Sciences, 9(22):4753.

Bardyn, J., Melly, T., Seller, O., and Sornin, N. (2016). Iot: The era of lpwan is starting now. In ESSCIRC, pages 25-30. 
Centenaro, M., Vangelista, L., Zanella, A., and Zorzi, M. (2016). Long-range communications in unlicensed bands: the rising stars in the iot and smart city scenarios. IEEE Wireless Communications, 23(5):60-67.

Farooq, M. O. and Pesch, D. (2018). Analyzing lora: A use case perspective. In 2018 IEEE 4th World Forum on Internet of Things (WF-IoT), pages 355-360.

Guibene, W., Nowack, J., Chalikias, N., Fitzgibbon, K., Kelly, M., and Prendergast, D. (2017). Evaluation of lpwan technologies for smart cities: River monitoring use-case. In IEEE WCNCW, pages 1-5.

Hattarge, S., Kekre, A., and Kothari, A. (2018). Lorawan based gps tracking of city-buses for smart public transport system. In ICSCCC 2018, pages 265-269.

James, J. G. and Nair, S. (2017). Efficient, real-time tracking of public transport, using lorawan and rf transceivers. In TENCON 2017, pages 2258-2261.

LoRa Alliance (2017). LoRaWAN 1.1 Specification. LoRa Alliance.

Oliveira, L. R., da Conceição, A. F., and Neto, L. P. S. (2018). Revisão sistemática da literatura sobre aplicações das tecnologias lora e lorawan. In SBESC.

Ortiz, F. M., Almeida, T. T., Ferreira, A. E., and Costa, L. H. M. K. (2019). Caracterização de desempenho de uma rede lora em ambientes urbanos: Simulação vs. prática. In $S B R C$, page 14.

Ortiz, F. M., Cruz, P., de S. Couto, R., and Costa, L. H. M. K. (2018). Caracterização de uma rede sem-fio de baixa potência e longo alcance para internet das coisas. In $S B R C$.

Podevijn, N., Plets, D., Trogh, J., Martens, L., Suanet, P., Hendrikse, K., and Joseph, W. (2018). Tdoa-based outdoor positioning with tracking algorithm in a public lora network. Wireless Communications \& Mobile Computing, page 9.

Ramli, N., Zabidi, M. M. M., Ahmad, A., and Musliman, I. A. (2019). Lora based vehicle tracking system. Indonesian Journal of Electrical Eng. and Informatics, 7(2).

Rossato, J. and Camargo, E. (2019). Implantação e Avaliação de uma Rede LoRaWAN na UTFPR - Câmpus Toledo. Trabalho de Conclusão de Curso, Tec. Sistemas para Internet, UTFPR, Toledo, Brasil.

Santana, E. F. Z., Chaves, A. P., Gerosa, M. A., Kon, F., and Milojicic, D. S. (2017). Software platforms for smart cities: Concepts, requirements, challenges, and a unified reference architecture. ACM Comput. Surv., 50(6).

Sánchez-Corcuera, R., Nuñez-Marcos, A., Sesma-Solance, J., Bilbao-Jayo, A., Mulero, R., Zulaika, U., Azkune, G., and Almeida, A. (2019). Smart cities survey: Technologies, application domains and challenges for the cities of the future. International Journal of Distributed Sensor Networks, 15(6):1550147719853984.

Yaqoob, I., Hashem, I. A. T., Mehmood, Y., Gani, A., Mokhtar, S., and Guizani, S. (2017). Enabling communication technologies for smart cities. IEEE Communications Magazine, 55(1):112-120.

Zanella, A., Bui, N., Castellani, A., Vangelista, L., and Zorzi, M. (2014). Internet of things for smart cities. IEEE Internet of Things Journal, 1(1):22-32. 